\title{
Immunogenicity of NS4b Dengue 3 Virus Mimotope Presented to the Immune System as Multiple Antigen Peptide System
}

\author{
Nevis Amin, ${ }^{1}$ Maritza Pupo, ${ }^{2}$ Alicia Aguilar, ${ }^{1}$ Frank Camacho, ${ }^{1}$ \\ Mayling Alvarez, ${ }^{2}$ Yamira Caballero, ${ }^{2}$ Daiyana Díaz, ${ }^{1}$ Angélica García, ${ }^{2}$ Osvaldo Reyes, ${ }^{3}$ \\ María Guadalupe Guzmán, ${ }^{2}$ David I. Stott, ${ }^{4}$ and Armando Acosta ${ }^{1}$ \\ ${ }^{1}$ Finlay Institute, calle 27, No. 19805, La Lisa, AP 16017, cod 11600 Havana, Cuba \\ ${ }^{2}$ Department of Virology, PAHO/WHO Collaborating Center for the Study of Dengue and Its Vector, \\ Pedro Kourí Tropical Medicine Institute (IPK), Autopista Novia del Mediodía Km 6, Apdo 601, Marianao 13, Havana, Cuba \\ ${ }^{3}$ Center for Genetic Engineering and Biotechnology (CIGB), Avenue 31, P.O. Box 6162, 10600 Habana, Cuba \\ ${ }^{4}$ Institute of Infection, Immunity and Inflammation, Glasgow Biomedical Research Centre, University of Glasgow, \\ 120 University Place, Glasgow G12 8TA, UK
}

Correspondence should be addressed to Nevis Amin; namin@finlay.edu.cu

Received 27 June 2013; Accepted 17 July 2013

Academic Editors: H. Imataka, P. Luksamijarulkul, M. Magnani, and S. Pöhlmann

Copyright (C) 2013 Nevis Amin et al. This is an open access article distributed under the Creative Commons Attribution License, which permits unrestricted use, distribution, and reproduction in any medium, provided the original work is properly cited.

The availability of random peptide libraries displayed on bacteriophage (RPL) has provided a powerful tool for selecting sequences that mimic binding properties of natural antigen epitopes (mimotopes). These mimotopes can be used for vaccine design, drug development, and diagnostic assays. Several mimotopes have been shown to induce production of antibodies against the natural antigen. We have previously identified four dengue virus mimotopes from a phage-displayed peptide library using antidengue 3 human sera. Three of them showed similarity in their amino acid sequences with the NS4b proteins of dengue. Few studies have examined the role of NS4b proteins in the antibody response to dengue virus infection. A multiple antigen peptide (MAP) system was chemically synthesized containing this mimotope (NS4b MAP), and BALB/c mice were immunized to evaluate its immunogenicity. Antipeptide responses were induced and recognised DENV-3 infected cells as determined by immunofluorescence. The high levels of the IgG2a subtype against NS4bMAP suggest the induction of a Thl-like response. Our findings suggest that the NS4b mimotope might be a useful tool for the development of multiepitope diagnostic assays, dengue virus vaccine design, and pathogenesis studies.

\section{Introduction}

Dengue is currently one of the most important mosquitoborne viral diseases of humans worldwide. Four viruses (dengue virus 1-4) belonging to the family Flaviviridae, genus Flavivirus, are responsible for this disease. Classically, two main syndromes are recognized: dengue fever and dengue hemorrhagic fever/dengue shock syndrome (DHF)/(DSS). The WHO classification of DHF/DSS was reviewed recently, and a new classification (dengue and severe dengue) has been proposed [1].

The dengue virus genome is composed of a linear, single stranded, and positive sense RNA molecule which is translated into a single polyprotein precursor comprising only one
ORF. The polyprotein is cotranslationally processed by host and virus specific proteases to 10 individual proteins: three structural proteins (capsid (C), premembrane (prM), and envelope (E)) and seven nonstructural (NS) proteins (NS1, 2a, 2b, 3, 4a, 4b, and 5) [2].

The identification and characterization of B-cell epitopes from dengue virus is highly relevant for understanding events related to natural infection, immunopathology, and for vaccines or diagnostic applications. B-cell epitopes have been reported from several dengue virus proteins, the greatest number being derived from $\mathrm{E}$ glycoprotein, followed by the nonstructural protein NS1 [3-5]. Although the C and prM/M proteins are conserved structural antigens, few B-cell epitopes have been defined from these proteins $[6,7]$. Linear epitopes 
from the small hydrophobic nonstructural NS4a and NS3 proteins have also been identified $[6,8,9]$.

Very little is known concerning the antigenic determinants of NS4b protein; it is involved in inhibition of the interferon response in host cells [10] and may also serve as an anchor for the viral replication complex to the endoplasmic reticulum membrane. Recently, it has been reported that dengue virus NS4b is primarily responsible for the induction of immunomediators such as IL6, IL-8, IP-10, TNF $\alpha$, and IFN $\gamma$ [11] and is a target for inhibition of dengue virus infection [12]. However the role of NS4b in the immune response has been poorly studied due to its transmembrane nature and the absence of an external surface domain.

Phage display has proven to be a powerful and economic technique for epitope identification and has been widely used in epitope mapping of Flavivirus [9]. We have previously identified four dengue mimotopes using a from a phage-displayed peptide library and showed similarity in their amino acid sequences with NS3 and NS4b nonstructural proteins of dengue virus [8]. The role of NS4b in the immune response has been poorly studied, but consistent antibody responses to NS4b in serum samples from dengue have been reported [13]. In order to determine whether NS4b can induce an antibody response against dengue virus, we synthesized a multiple antigen peptide system (MAP) containing the NS4b mimotope and tested its ability to stimulate an immune response. The knowledge derived from this study will help elucidate whether NS4b is involved in humoral immunity during the viral infection cycle.

\section{Materials and Methods}

2.1. Synthetic Peptides. A peptide construct was prepared in the synthetic peptide laboratory at the Center for Genetic Engineering and Biotechnology (CIGB, Havana City). Synthesis was performed manually on Fmoc-AM-MBHA resin by a stepwise solid-phase procedure using the Fmoc/tBu strategy and contained NS4b mimotope: FERVPGEV [8]. The peptide was characterized by reversed phase high-performance liquid chromatography and electrospray ionization mass spectrometry. A four-armed MAP based on the NS4b mimotope sequence was synthesized (Figure 1) and named as NS4b MAP.

Additional peptides to be used as controls in all assays were also synthesized. A positive control peptide (E, mimetic of envelope dengue protein), was derived from screening a phage-displayed peptide library with the $\mathrm{H} 3 / 6$ monoclonal antibody [14]; a negative control peptide, MAP46-56, containing two HAV mimotopes (46 and 56) was previously described by Larralde et al. [15].

2.2. Polyclonal Antibodies. NS4b-specific polyclonal rabbit antiserum, raised against a GST-NS4b 127-170 aa fusion protein, was a generous gift from Dr. Ralf Bartenschlager, Department of Molecular Virology, University of Heidelberg, Germany [10].

2.3. Purification of Phages Displaying Peptide Mimetic NS4b. Phages clones $(\mathrm{Ph}) 2,15$, and $35[8]$ and M13 K07 phage

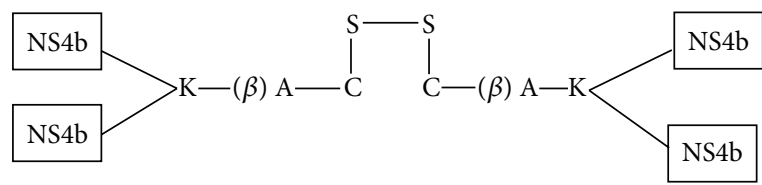

NS4b FERVPGEV

Figure 1: Chemical structure of MAP of NS4b mimotope sequence.

(wild type control) were amplified in liquid cultures of E. coli TG1 and purified by precipitation with polyethylene glycol (PEG8000)/NaCL. Each purified phage clone was tittered, and its concentration was adjusted to $10^{12} \mathrm{UFC} / \mathrm{mL}$ for ELISA assays [15].

2.4. Anti-NS4b Polyclonal Antibody ELISA. Recognition of phage clones and peptide constructs by dengue anti-NS4b polyclonal antiserum was evaluated by an indirect ELISA. Multiwell plates (Nunc Maxisorp F8, Life Technologies Limited, Paisley, UK) were coated with purified phages $\left(10^{8} \mathrm{CFU} /\right.$ well) and peptides $(5 \mu \mathrm{g} / \mathrm{mL})$ diluted in $0.05 \mathrm{M}$ sodium carbonate buffer $\mathrm{pH} 9.6$ (coating buffer), during $18 \mathrm{~h}$ at $4^{\circ} \mathrm{C}$. Plates were washed thrice with phosphate buffered saline in 0.05\% Tween 20 (PBS/T) and blocked with skimmed milk $(3 \% \mathrm{w} / \mathrm{v})$ in $\mathrm{PBS} / \mathrm{T}$ (blocking solution). Rabbit anti-NS4b polyclonal antiserum diluted in blocking solution $(1: 500$, $100 \mu \mathrm{L}$ per well) was added to the plates for $1 \mathrm{~h}$ at $37^{\circ} \mathrm{C}$. After repeating the washes, bound antibodies were detected with HRP-conjugated goat anti-rabbit IgG antibodies (Sigma-Aldrich, UK), $\mathrm{H}_{2} \mathrm{O}_{2}$, and orthophenylenediamine (OPD). The reaction was stopped with $0.1 \mathrm{M} \mathrm{H}_{2} \mathrm{SO}_{4}$, and the absorbance read at $492 \mathrm{~nm}$ in an ELISA plate reader. All samples were separately tested and by duplicate, and results were expressed as mean values. Phage M13 K07, MAP46-56, and $\mathrm{E}$ peptide were used as controls in all assays.

2.5. Immunization of Mice. Groups of 10, 4-6 weeks old, male $\mathrm{BALB} / \mathrm{c}$ mice (from the Center for Production of Laboratory Animals CENPALAB, Havana city Cuba) were injected intraperitoneally with $50 \mu \mathrm{g}$ of NS4b MAP on days 0,14 , 35 , and 56 . The antigens were emulsified with, the complete Freund adjuvant in the first injection and the incomplete Freund adjuvant in successive ones in a final volume of $0.2 \mathrm{~mL}$. Similarly, negative control mice received four doses of adjuvant alone. Serum samples were obtained from mice 15 days after the last boost and stored at $-20^{\circ} \mathrm{C}$ until use. All animal experimentation was carried out in compliance with the legal requirements of the national authority.

2.6. Serum ELISA to Detect Anti-Peptide Antibodies. Peptide recognition by anti-peptide antibodies in serum samples from groups of immunized animals was determined by an indirect ELISA. Multiwell plates (Nunc Maxisorp F8, Life Technologies Limited, Paisley) were coated with peptide constructs $(20 \mu \mathrm{g} / \mathrm{mL}$ in $50 \mathrm{mM} \mathrm{NaHCO} 3, \mathrm{pH} 9.6)$. Plates were incubated overnight at $4^{\circ} \mathrm{C}$, then washed 3 times with PBS$\mathrm{T}$, and blocked with PBS-T/3\% bovine serum albumin (BSA) for $1 \mathrm{~h}$ at $37^{\circ} \mathrm{C}$. Serum samples (1/50 dilution in PBS-T/BSA) from each group were added in duplicate $(100 \mu \mathrm{L} /$ well $)$ and 
incubated $2 \mathrm{~h}$ at $37^{\circ} \mathrm{C}$. Plates were washed 3 times with PBS$\mathrm{T}$, then incubated for $1 \mathrm{~h}$ at $37^{\circ} \mathrm{C}$ with $100 \mu \mathrm{L} /$ well of goat anti-mouse IgG/HRP (Sigma-Aldrich, UK, diluted 1:5000), washed, and developed for $20 \mathrm{~min}$ with OPD (Sigma, UK). The reaction was stopped with $100 \mu \mathrm{L}$ of $0.1 \mathrm{M} \mathrm{H}_{2} \mathrm{SO}_{4}$, and the absorbance at $492 \mathrm{~nm}$ was recorded by an automated ELISA reader (Dynex Technologies, UK).

Control ELISAs using the E peptide as capture antigen were run in parallel. All serum samples were analyzed in duplicate, and mean values were used for analysis. Each ELISA was performed twice. Results were expressed as the ratio of ELISA OD492 nm of immune sera/OD492 nm of preimmune sera. A ratio of $\geq 2$ with respect to control groups was chosen as the cutoff value to consider the serum sample as positive.

Antibody isotypes IgG1 and IgG2a were assayed similarly, but using pooled sera from each group of mice, diluted 1/50. The corresponding goat anti-mouse isotype-specific antibody was used (SIGMA, USA), and the reactivity was revealed with HRPO-conjugated rabbit anti-goat IgG antibody (SIGMA, USA).

2.7. Immunofluorescence Assay (IFA). The Aedes albopictus cell line (clone $\mathrm{C} 6 / 36-\mathrm{HT}$ ) was grown at $33^{\circ} \mathrm{C}$ in MEM medium containing $2 \mathrm{mmol} / \mathrm{L}$ glutamine and $10 \%$ heat-inactivated fetal bovine serum (FBS). For the assay, they were maintained in the same medium with $2 \%$ of FBS.

Viruses. Den-3 116/00 strain with a history of five passages in C6/36-HT cells (5p C636) and one passage in Vero cells (1p Vero) was employed at a titer of $1.2 \times 10^{5} \mathrm{PFU} / \mathrm{mL}$.

Immunofluorescence Staining. Anti-DEN-3 virus antibodies in immune sera were detected by indirect immunofluorescence. C6/36-HT cells were inoculated with Den-3 116/00 (5p C636, 1p Vero) (m.o.i.0.01) following the shell vial method described by Roche et al. [16]. The cells were harvested from $10 \mathrm{~h}$ to $48 \mathrm{~h}$ postinfection (p. i.), fixed on slides for $20 \mathrm{~min}$ in ice cold acetone and incubated with mouse serum diluted $1: 20$ in PBS. Binding of mouse immunoglobulin to cells was detected using fluorescein-isothiocyanate (FITC) conjugated goat anti-mouse IgG antibody (Sigma) and examined under a fluorescence microscope (Leitz Wetzler Germany).

The NS4b-specific polyclonal antiserum was used as a positive control in all assays. The NS4b protein expressed in infected cells was detected by immunofluorescence staining using the NS4b specific polyclonal antiserum at 1: 20 dilution in phosphate buffered saline (PBS) as the primary antibody and FITC-conjugate goat anti-rabbit IgG (Sigma) diluted 1:40 in PBS as the secondary antibody.

Pooled serum from preimmune mice was included as negative control.

All photographs were taken at the same magnification.

2.8. Statistical Analysis. The Mann-Whitney test was used to compare the results obtained in serum samples from immunized mice and preimmune sera and was considered significant if $P<0.05$.

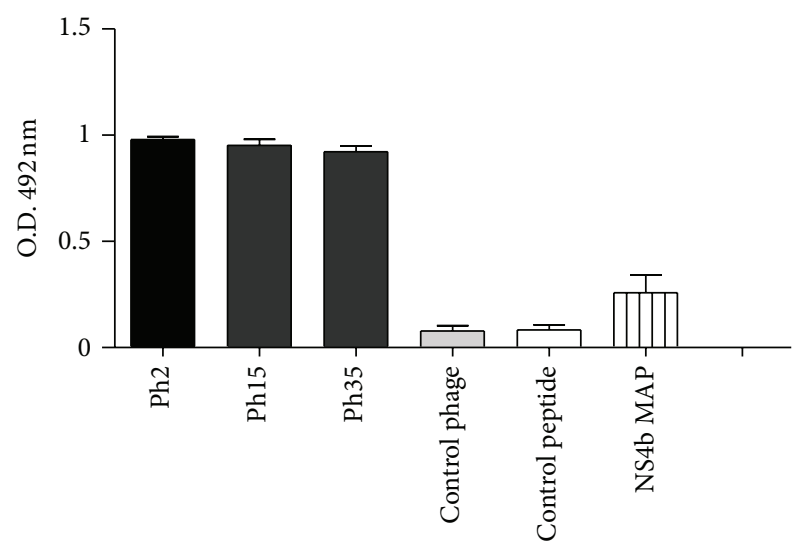

FIGURE 2: ELISA reactivity with NS4b specific polyclonal antiserum of phage clones 2,15 , and 35 selected from a phage displayed random peptide library, M13K07 wild type control, and NS4b MAP. E peptide was used as a negative control. The bars represent SDs. The data represent the average of two separate experiments.

\section{Results}

3.1. NS4b Mimotope Characterization Using the NS4b-Specific Polyclonal Antiserum. An NS4b MAP (Figure 1) was designed according to our previous results [8]. To verify the antigenic similarity of the phage-displayed peptide and MAP construct, their capacity to bind to the NS4b specific polyclonal antiserum was evaluated in an ELISA. As shown in Figure 2 the NS4b polyclonal antiserum reacted with all the phage clones and NS4b MAP. In contrast, the antiserum did not bind significantly to the control peptides or the control phage clone M13 K07 (Figure 2), showing that NS4b mimotope exhibits structural similarity with the dengue virus epitope.

\subsection{Anti-Peptide Antibody Responses and Isotype Distribution} in Immunized Mice. The immunogenicity of the NS4b MAP was evaluated by measuring peptide specific IgG antibody levels in serum from immunized mice. The sera were assayed individually against NS4b MAP as the capture antigen using an ELISA assay. NS4b MAP induced significant antipeptide IgG levels $(P<0.05)$ (Figure 3$)$. Preimmune sera did not display ELISA reactivity.

The NS4b-specific IgG2a response was higher than IgG1 levels indicating a predominantly $\mathrm{T}_{\mathrm{h}} 1$-type response (Figure 4).

This assay illustrated that NS4b MAP elicits IgG2a antiNS4b MAP antibodies in immunized mice; however it does not indicate that these would bind infectious DEN-virus. To determine if these antibodies possess the capacity to recognize DEN-virus, the antiviral antibody response was studied.

3.3. Specific Response of Antiserum from Mice Immunized with NS4b MAP. Induction of an antidengue response by NS4b MAP at 56 days postimmunization was tested in individual mice. Specific anti-dengue antibodies were detected by IFA in sera from mice immunized with the NS4b MAP compared with control sera. Four sera from 5 immunized mice 


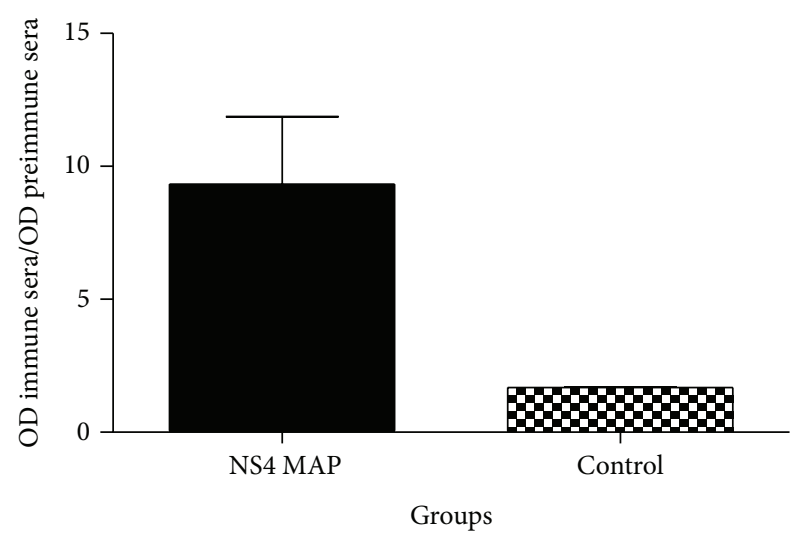

FIGURE 3: Anti-peptide antibody levels elicited by NS4b mimotope as MAP. Serum samples were obtained on day 0 (preimmune) and after four injections (day 56) from mice immunized with $50 \mu \mathrm{g}$ of antigen (NS4b MAP). The reactivity of each serum was individually tested, and the average of the results is shown. ELISA plates were coated with the homologous peptide, and sera were diluted 1:50. Results are expressed as OD ratio and are presented for OD492 nm (day 56)/OD492 nm (preimmune sera). The cutoff value is an OD ratio $\geq 2.0$. Antibody levels against NS4b as MAP were significantly higher $(P<0.05)$ than the PBS control.

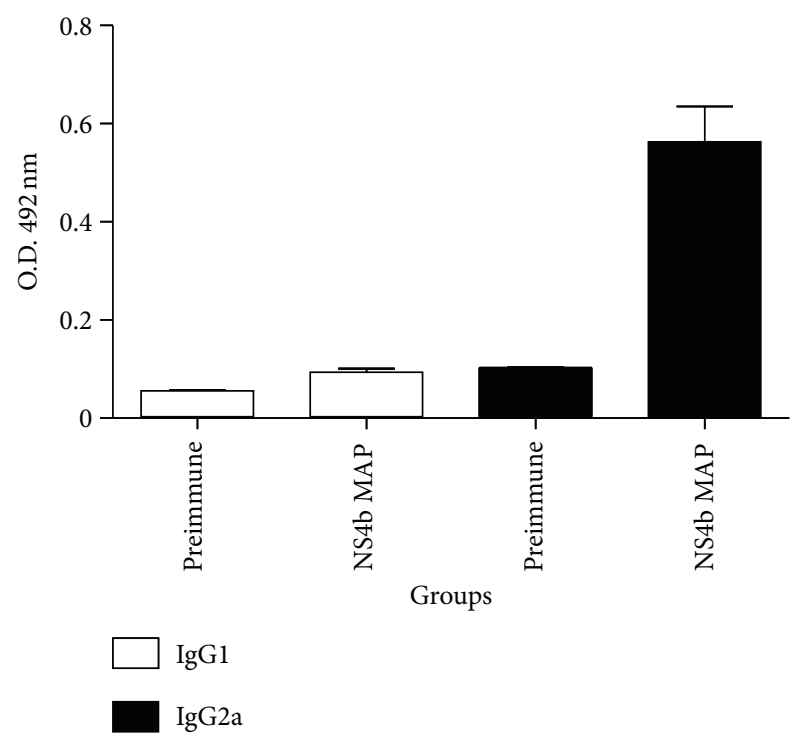

FIGURE 4: IgG1 and IgG2a subclasses induced against NS4b mimotope as MAP after three immunizations (mean from three determinations). Pooled sera from each group of mice $(n=10)$ were diluted $1: 20$. Plates were developed using isotype-specific reagents.

reacted with DENV-3 expressed in C636 HT cells, whereas no immunofluorescence was observed in noninfected cells (Figure 5(d)). Fluorescent reactivity was not detected in sera from preimmune mice.

Kinetic expression of viral antigens in infected cells was also analysed with pooled immune sera. Weak positive staining was observed in the cytoplasm at $10 \mathrm{~h}$ p.i.; by $12 \mathrm{~h}$ the signal became more intense with punctuated distribution mainly in the perinuclear region of the cytoplasm (Figure 5(a)). Between $16 \mathrm{~h}$ and $20 \mathrm{~h}$ postinfection, the infected cells produced a strong cytoplasmic staining pattern (Figure 5(b)), with the highest intensity at $24 \mathrm{~h}$ p.i. (Figure 5(c)). The intensity of the signal declined from $48 \mathrm{~h}$ p.i.

Anti-NS4b positive control serum detected the expression of NS4b protein of DENV-2 (data not shown) as reported previously by Miller et al. [10].

\section{Discussion}

Synthetic peptides derived from mimotopes have been used to induce specific immune responses against proteins [17]. They have several advantages as immunogens, including simple antigenic composition, low cost, ease of manufacture, control of production scale, absence of risk of reversion to the wild-type, virulent form, and better stability compared with other vaccine technologies. Presentation of synthetic peptides as MAPs has been shown to be valuable in vaccine development $[3,7,18,19]$; since the increased molecular weight and repeating peptide structure of MAPs have been shown to improve immunogenicity [20].

B-cell epitopes have been identified on both structural and nonstructural dengue proteins including E, NS1, NS4a, prM, and NS3 [21]. However, the antigenic and immunogenic characteristics of NS4b protein of dengue virus have been poorly explored. We have previously identified a DENV-3 phage mimotope with anti-dengue antibodies in human sera; this mimotope showed partial homology with NS4b dengue protein (164-171 aa region) [8]. Recently we have demonstrated that NS4b MAP was recognized by sera from patients infected with all the serotypes of dengue virus, the highest reactivity being with sera from DENV-3 infected patients. In the present study we evaluated the immunogenicity of NS4b mimotope presented to the immune system as a MAP construct.

Previously studied dengue phage mimotopes ( $\mathrm{Ph} 2, \mathrm{Ph} 15$, and Ph35) share sequence similarity (FERVPGEVT) and partial sequence homology (four residues) with amino acids 164-171 of the NS4b dengue protein [8]. This region is highly conserved among dengue viruses and was described as a transmembrane domain by Miller et al. [10]. The evaluation of similarities in the physicochemical properties of nonhomologous amino acid residues could be important in the structural analysis of linear sequences. Figure 6 shows that the NS4b mimotope differs from the NS4b sequence by four amino acids (R166 K, V167Q, P168L, and E169Q), and these changed residues show similarities in the size, polarity, net charge, or aliphatic character. These physicochemical similarities could result in the peptide adopting a conformation homologous with the dengue virus epitope, which would explain the recognition of the cloned peptides by anti-dengue antibodies, despite the absence of complete sequence homology with the original protein.

Antigenic mimicry among the NS4b epitope, phage mimotopes, and NS4b MAP was determined by binding to NS4b-specific polyclonal antiserum. Phage mimotopes and NS4b MAP reacted with the antiserum, reflecting the antigenic similarity between the mimotope peptide and NS4 Bcell epitope. While several studies, including those of our 


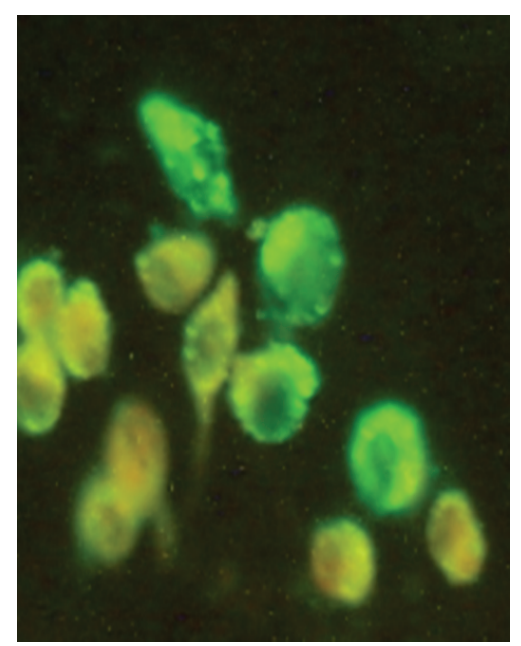

(a)

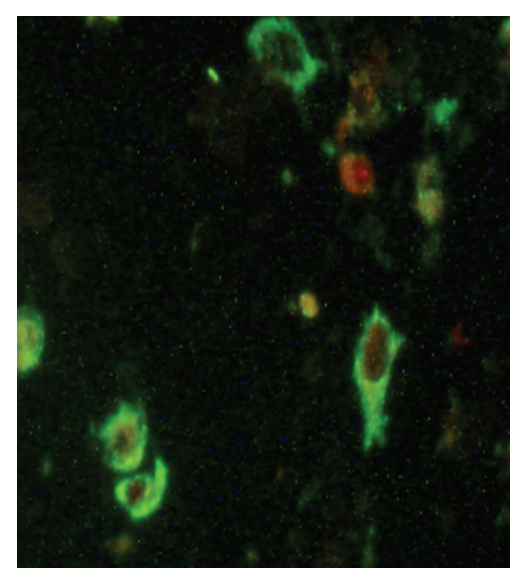

(c)

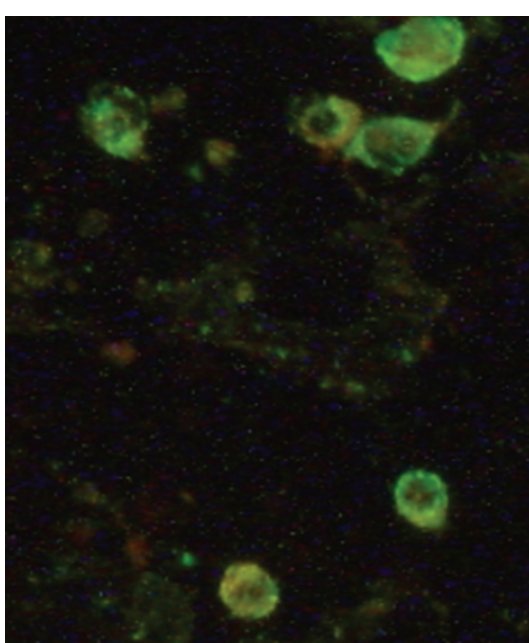

(b)

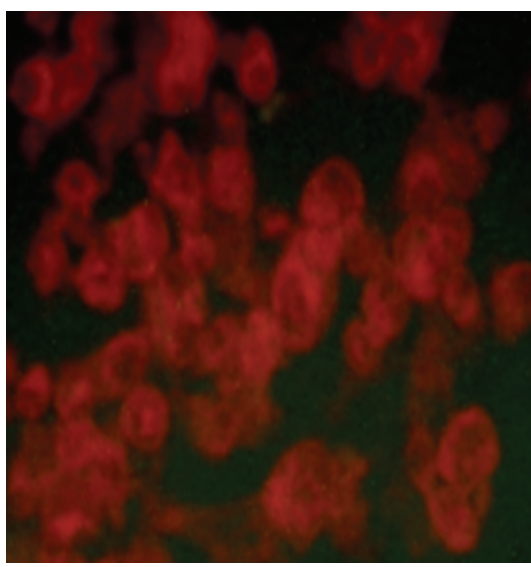

(d)

FIGURE 5: Immunofluorescence staining of DEN-infected cells. C636 HT cells were infected with Den-3 116/00 (5p C636, 1p VERO) (m.o.i.0.01). At 12 (a), 20 (b), and $24 \mathrm{~h}$ p.i. (c), the infected cells reacted with individual serum from mice immunized with $50 \mu \mathrm{g}$ of antigen (NS4b MAP). Non-infected C6/36 HT cells were used as a negative control (d).

laboratory with HAV mimotopes (personal communication), found that the antigenicity and immunogenicity of a peptide mimotope can vary by changing the molecular context in which the peptide is first identified [17], the NS4b mimotope was still able to bind dengue virus specific antisera and elicit antibodies binding to dengue virus when removed from the filamentous phage context, where this mimotope was first identified.

The ability of peptide mimotopes selected from phage displayed, random peptide libraries to induce specific immune responses has been reported previously $[9,16,17]$. In this study a four-armed MAP containing the NS4b mimotope sequence of DENV-3 was used as immunogen to stimulate antibodies against dengue virus. The MAP approach has also been used in the preparation of experimental subunit vaccines against different pathogens, and has multiple advantages $[3,7,17,18]$. In ours hands, NS4b MAP was immunogenic in BALB/c mice, and the elicited antibodies specifically recognised DENV-3 expressed in cells infected with strain Den-3 116/00 by immunofluorescence. Although IF against

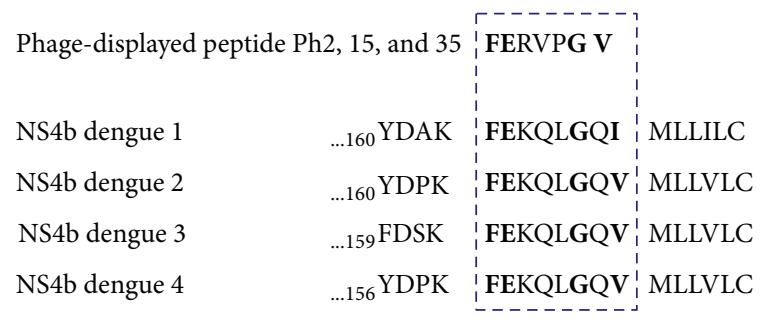

FIGURE 6: Alignment of the mimotope cloned from phages $\mathrm{Ph} 2,15$, and 35 with NS4B protein.

the others serotypes was not tested, it is believed that a similar behaviour could occur, since this small region of NS4b protein is highly conserved among the four serotypes of dengue (Figure 6). Similarly, Tian et al. [9] identified a new conservative and continuous epitope of DENV-2 NS3 protein using a phage-displayed peptide library and demonstrated its potential application in the design of an epitope vaccine to prevent dengue infection. 
It is interesting to note that topological studies of NS4B show that the sequence homologous with the mimotope (aa 164-171) seems to reside in the transmembrane region of the endoplasmic reticulum [10]. Our results indicate that the NS4b epitope could be accessible for binding to B-cell receptors during cell lysis induced by the virus or immune cell mediated lysis [22]. Another explanation could be that dendritic cells, monocytes/macrophages, and B cells are target cells for dengue virus, and NS4b protein produced in the cytoplasm of these infected cells during the cycle of replication could be degraded into peptides that enter in the class II MHC pathways of antigenic presentation.

The kinetic expression of dengue NS4b protein has previously been studied in infected cells [10]. We detected the protein from $10 \mathrm{~h}$ postinfection using pooled sera from mice at 56 days postimmunization, and similar results were observed with the antiNS4b specific polyclonal antiserum. These results suggest that the antibodies elicited by NS4b mimotope are able to recognize NS4b protein, which is produced in the first step of viral replication.

T-cell responses were studied by measuring IgG subclass composition of sera from immunized mice. The highest levels of the anti-NS4b IgG2a subtype suggests the induction of a $T_{h} 1$-like response. $A T_{h} 1$ response with the expression of IgG2a would be desirable, as this murine isotype has been reported to be particularly effective for virus clearance. Furthermore, $\mathrm{T}_{\mathrm{h}} 1$ cells may contribute to protection against dengue by upregulating the cytotoxic response to destroy virus infected cells early in infection [23]. The signature cytokine of $T_{h} 1$ cells is INF $\gamma$ the most potent macrophage-activating cytokine in the immune system. This cytokine has been described as a mediator of the cellular immune response and plays a role in the antiviral activity against DENV [24]. Further studies are needed to determine the secretion of this cytokine by stimulated $\mathrm{CD}^{+}$and $\mathrm{CD} 8^{+} \mathrm{T}$ cells from mice immunized with NS4b MAP.

The antibody response generated by NS4b MAP could indicate the presence of both $\mathrm{T}$ and $\mathrm{B}$ epitopes in the region amino acids $164-171$ of the NS4b dengue protein sequence.

\section{Conclusions}

In the present study, we evaluated the antigenicity and immunogenicity of a NS4b dengue virus mimotope. The MAP construct containing four arms of this mimotope induced anti-peptide antibodies that specifically recognised DENV3 expressed in infected cells. Our results suggest that the NS4b peptide could be included as part of an experimental antidengue subunit vaccine. These findings also suggest that the NS4b protein of dengue virus could be implicated in the humoral response.

\section{Acknowledgments}

The authors are grateful to Dr. Ralf Bartenschlager and Wolfgang Fischl, Department of Molecular Virology, University of Heidelberg for their generous gift of the anti-NS4b polyclonal antibody used in their experiments. they also thank Virginia
Capo for assistance in the photographs of the immunofluorescence staining.

\section{References}

[1] M. G. Guzman, L. Hermida, L. Bernardo, R. Ramirez, and G. Guillen, "Domain III of teh envelope protein as a dengue vaccine target," Expert Review of Vaccines, vol. 9, no. 1, pp. 87-97, 2010.

[2] B. D. Lidenbach and C. M. Rice, "Flaviviridae: the viruses and their replicaction," in Fields Virology, D. M. Knipe and P. M. Howley, Eds., pp. 991-1041, Lippincott Williams and Wilkins, Philadelphia, Pa, USA, 4th edition, 2001.

[3] G. Amexis and N. S. Young, "Multiple antigenic peptides as vaccine platform for the induction of humoral responses against dengue-2 virus," Viral Immunology, vol. 20, no. 4, pp. 657-663, 2007.

[4] A. N. M. R. da Silva, E. J. M. Nascimento, M. T. Cordeiro et al., "Identification of continuous human B-cell epitopes in the envelope glycoprotein of dengue virus type 3 (DENV-3)," PloS ONE, vol. 4, no. 10, Article ID e7425, 2009.

[5] L. Jiang, J.-M. Zhou, Y. Yin, D.-Y. Fang, Y.-X. Tang, and L.-F. Jiang, "Selection and identification of B-cell epitope on NS1 protein of dengue virus type 2," Virus Research, vol. 150, no. 1-2, pp. 49-55, 2010.

[6] R. AnandaRao, S. Swaminathan, and N. Khanna, "The identification of immunodominant linear epitopes of dengue type 2 virus capsid and NS4a proteins using pin-bound peptides," Virus Research, vol. 112, no. 1-2, pp. 60-68, 2005.

[7] S. Vázquez, M. G. Guzmán, G. Guillen et al., "Immune response to synthetic peptides of dengue prM protein," Vaccine, vol. 20, no. 13-14, pp. 1823-1830, 2002.

[8] N. Amin, A. Aguilar, F. Chamacho et al., "Identification of dengue-specific B-cell epitopes by phage-display random peptide library," Malaysian Journal of Medical Sciences, vol. 16, no. 4, pp. 4-14, 2009.

[9] Y. Tian, W. Chen, Y. Yang et al., "Identification of B cell epitopes of dengue virus 2 NS3 protein by monoclonal antibody," Applied Microbiology and Biotechnology, vol. 97, no. 4, pp. 1553-1560, 2013.

[10] S. Miller, S. Sparacio, and R. Bartenschlager, "Subcellular localization and membrane topology of the dengue virus type 2 nonstructural protein 4B," Journal of Biological Chemistry, vol. 281, no. 13, pp. 8854-8863, 2006.

[11] J. F. Kelley, P. H. Kaufusi, E. M. Volper, and V. R. Nerurkar, "Maturation of dengue virus nonstructural protein $4 \mathrm{~B}$ in monocytes enhances production of dengue hemorrhagic fever-associated chemokines and cytokines," Virology, vol. 418, no. 1, pp. 27-39, 2011.

[12] X. Xie, Q.-Y. Wang, H. Y. Xu et al., "Inhibition of dengue virus by targeting viral NS4B protein," Journal of Virology, vol. 85, no. 21, pp. 11183-11195, 2011.

[13] L. Lázaro, G. Mellado, J. García, A. Escobar, L. Santos, B. Gutiérrez et al., "Analysis of antibody responses in human dengue patients from the mexican coast using recombinant antigens," Vector Borne and Zoonotic Diseases, vol. 8, pp. 69-79, 2008.

[14] M. Pupo-Antúnez, H. Rodríguez, S. Vázquez et al., "Monoclonal antibodies raised to the Dengue-2 virus (Cuban: A15 strain) which recognize viral structural proteins," Hybridoma, vol. 16, no. 4, pp. 347-353, 1997.

[15] O. G. Larralde, R. Martinez, F. Camacho et al., "Identification of hepatitis A virus mimotopes by phage display, antigenicity and 
immunogenicity," Journal of Virological Methods, vol. 140, no. 1-2, pp. 49-58, 2007.

[16] R. R. Roche, M. Alvarez, M. G. Guzman, L. Morier, and G. Kouri, "Comparison of rapid centrifugation assay with conventional tissue culture method for isolation of dengue 2 virus in C6/36-HT cells," Journal of Clinical Microbiology, vol. 38, no. 9, pp. 3508-3510, 2000.

[17] M. Houimel and K. Dellagi, "Peptide mimotopes of rabies virus glycoprotein with immunogenic activity," Vaccine, vol. 27, no. 34, pp. 4648-4655, 2009.

[18] H. Garay, T. Menéndez, Y. Cruz-Leal et al., "Study of various presentation forms for a peptide mimetic of Neisseria meningitidis serogroup B capsular polysaccharide," Bioconjugate Chemistry, vol. 22, no. 1, pp. 33-41, 2011.

[19] B. Mahajan, J. A. Berzofsky, R. A. Boykins et al., "Multiple antigen peptide vaccines against Plasmodium falciparum malaria," Infection and Immunity, vol. 78, no. 11, pp. 4613-4624, 2010.

[20] J. P. Tam, "Synthesis and applications of branched peptides in immunological methods and vaccine," in Peptide: Synthesis, Structures and Applications, B. Gutte, Ed., pp. 455-500, Academic Press, New York, NY, USA, 1995.

[21] K. Vaughan, J. Greenbaum, M. Blythe, B. Peters, and A. Sette, "Meta-analysis of all immune epitope data in the flavivirus genus: inventory of current immune epitope data status in the context of virus immunity and immunopathology," Viral Immunology, vol. 23, no. 3, pp. 259-284, 2010.

[22] A. L. Rothman, "Immunity to dengue virus: a tale of original antigenic sin and tropical cytokine storms," Nature Reviews Immunology, vol. 11, no. 8, pp. 532-543, 2011.

[23] S. Li, L. Peng, W. Zhao et al., "Synthetic peptides containing Band T-cell epitope of dengue virus-2 E domain III provoked Band T-cell responses," Vaccine, vol. 29, no. 20, pp. 3695-3702, 2011.

[24] K. Clyde, J. L. Kyle, and E. Harris, "Recent advances in deciphering viral and host determinants of dengue virus replication and pathogenesis," Journal of Virology, vol. 80, no. 23, pp. 1141811431, 2006. 

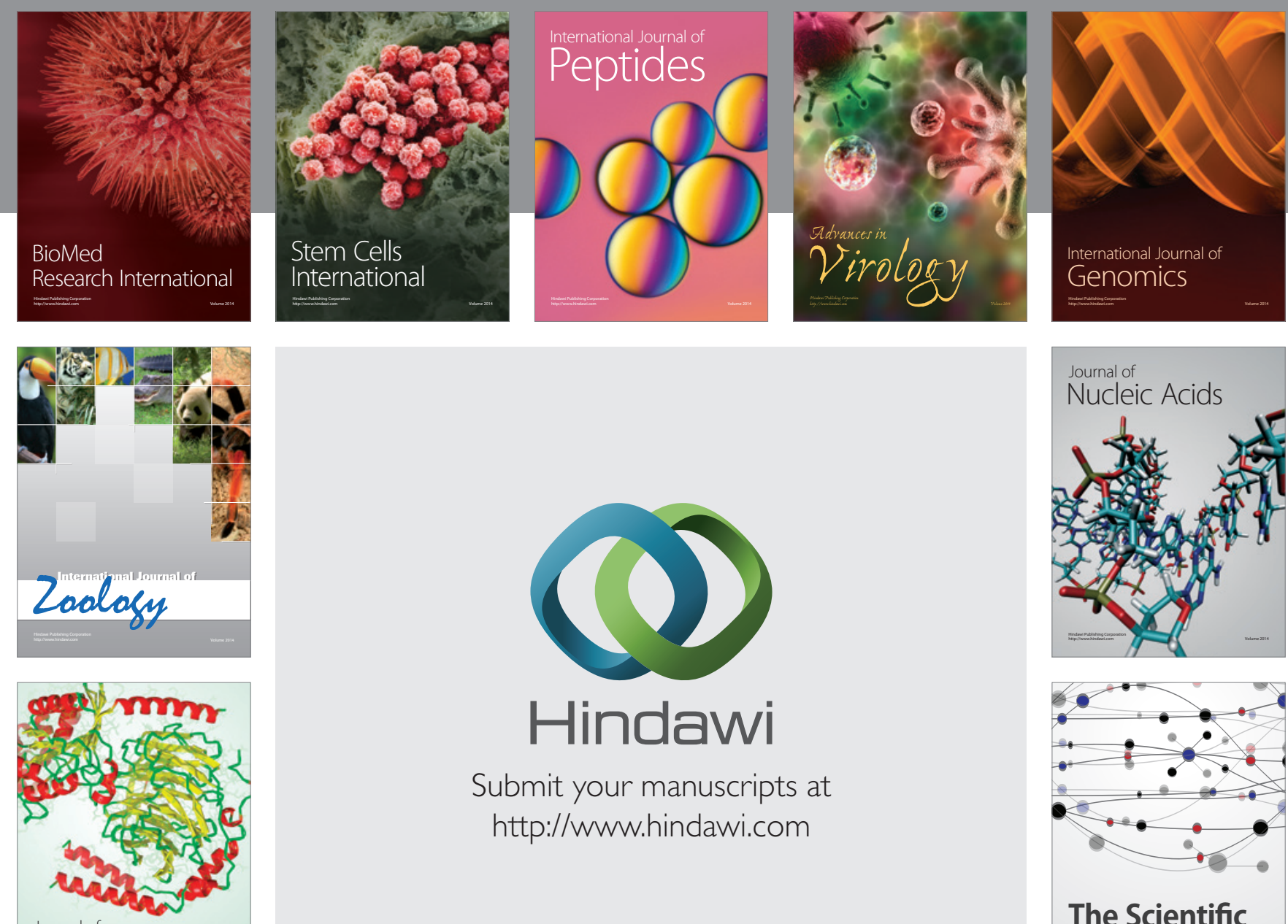

Submit your manuscripts at

http://www.hindawi.com

Journal of
Signal Transduction
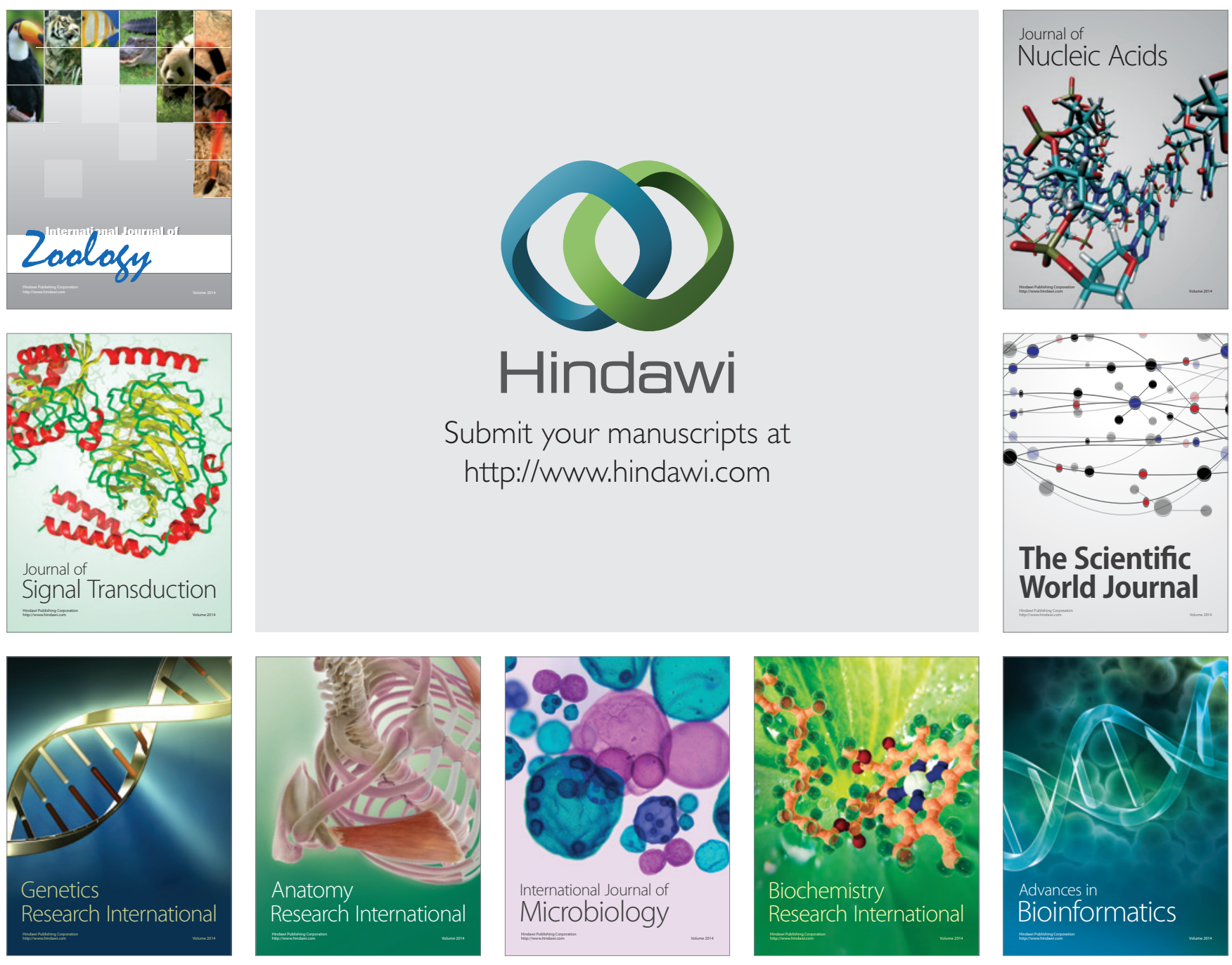

The Scientific World Journal
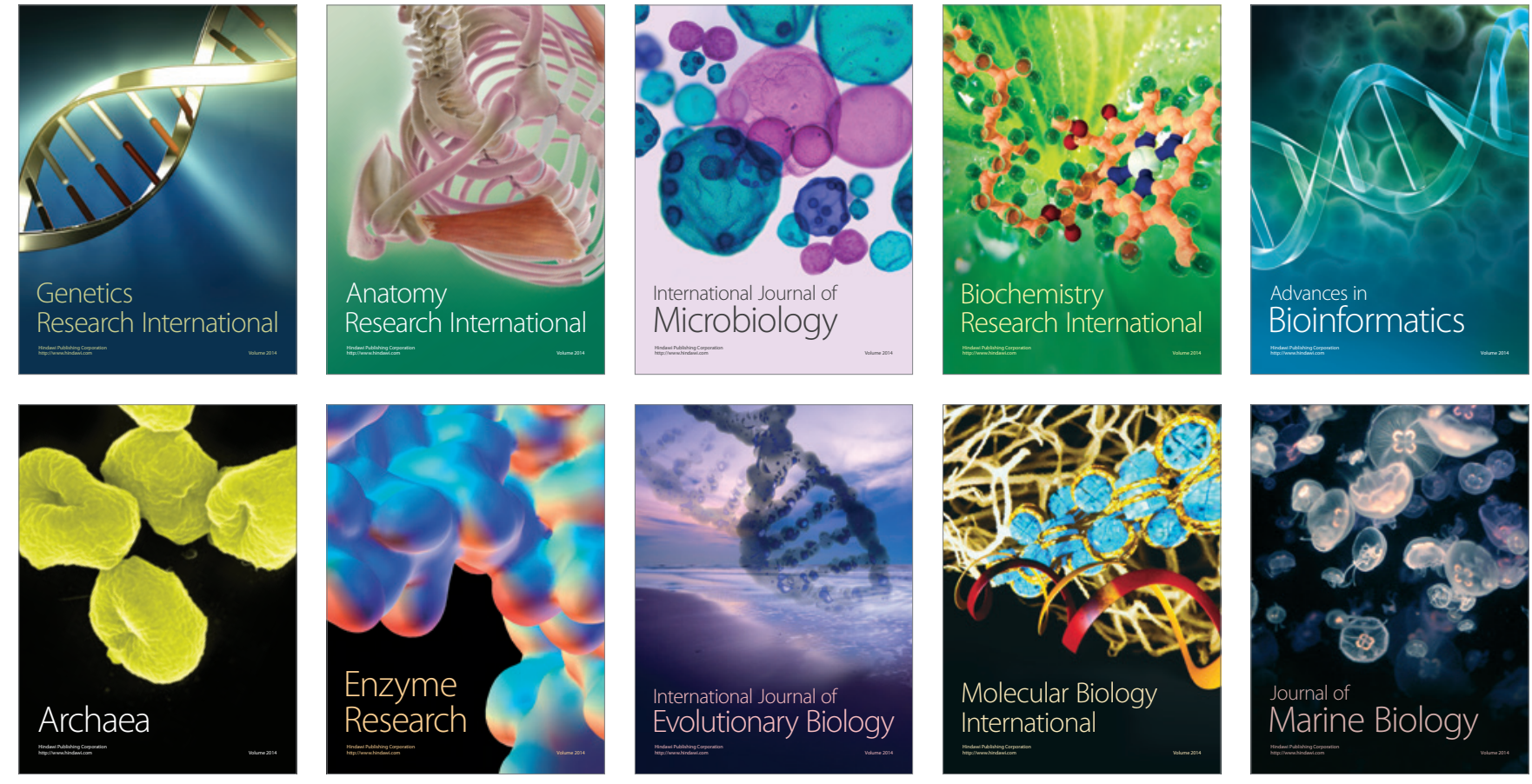